\title{
Monodromy Representations for Generalized Knizhnik-Zamolodchikov Equations and Hecke Algebras
}

\author{
By \\ Ivan CHEREDNIK*
}

\begin{abstract}
In the paper generalizations of the Knizhnik-Zamolodchikov equation with symmetries in Weyl groups for arbitrary classical $r$-matrices are studied. Their monodromy representations are calculated explicitly in terms of Hecke algebras and affine Hecke algebras for basic cxamples.
\end{abstract}

\section{§0. Introduction}

The aim of this paper is to generalize the so-called Knizhnik-Zamolodchikov differential equation for $n$-point function from the two-dimensional conformal field theory [1] and to determine the corresponding monodromy representation. We develop the results of Tsuchiya, Kanie [2], Kohno [3,4] and Drinfeld (concerning the $\mathrm{KZ}$-equation and its natural version with the values in simple $\mathbb{L i e}$ algebras) for some new $W$-invariant equations $[5,6]$ connected with classical $W$-invariant $r$-matrices for arbitrary Weyl groups $W$. Monodromy matrices are calculated explicitly for classical $W$ and some special $r$-matrices (in the fundamental representation of $g \ell_{N}$ ) by means of Hecke algebras of corresponding types.

In general, one obtains certain representations of Birman-Wenzl algebras or some their generalizations of the same type as $W$.

Our main result on the monodromy (Theorem 3) is, however, formulated in terms of the affine Hecke algebra $\mathscr{H}_{n}^{q}$ for $G L_{n}$ and not by means of Hecke algebras of type $A_{n-1}, B_{n}, C_{n}$. There is no contradiction with the above general statement since the latter are some quotient algebras of $\mathscr{H}_{n}^{q}$. We do not use $\mathscr{H}_{n}^{q}$ for the sake of uniformity only. This very algebra plays a key role in the theory of Young's bases for Weyl groups and Hecke algebras of classical types.

We follow [5,6] and determine explicitly Young's bases for irreducible "semi-simple" representations of some extention of $\mathbb{C}[W]$ (for classical $W$ ). In the special case $W=S_{n}$ this extension is the so-called degenerate $(q \rightarrow 1)$ affine

Communicated by M. Kashiwara, April 3, 1990.

1991 Mathematics Subject Classification: 11F60, 33D70.

* A.N. Belozersky Laboratory, Bldg. "A", Moscow State University, Moscow 119899, USSR. 
Hecke algebra $\mathscr{H}_{n}^{\prime}$ (Murphy, Drinfeld, Lusztig). We define the KZ-equation for this algebra and calculate its monodromy, which turns out to be a representation of $\mathscr{H}_{n}^{q}$.

There are some applications. The monodromy gives us an isomorphism $\mathscr{H}_{n}^{q} \widetilde{\rightarrow} \mathscr{H}_{n}^{\prime}$ (for general $q$ ), constructive isomorphisms between $C[W]$ and corresponding Hecke algebras and a regular method of "exponentiating" of quantum Yang's $R$-matrices to their trigonometric counterparts. Moreover, one obtains a way of realization of any irreducible representation for $\mathcal{H}_{n}^{q}$ as a monodromy representation for a certain local system and gets some new invariants of links in $\mathbb{R}^{3}$ without a circle $S^{1}$ (or two circles for affine $W$ ). All these results are connected with Kazhdan, Lusztig, Ginsburg et al. approach to Hecke algebras from the point of view of the Langlands-Deligne conjecture. We arrive, in fact, at some special version of this conjecture with the monodromy group (or $\left.\mathcal{H}_{n}^{q}\right)$ as the Galois group and representations of $C[W]$ or corresponding Hecke algebras in place of $p$-adic representations.

\section{\$1. W-invariant Local Systems}

Let $R=\{\alpha\}$ be a root system of type $X_{n}=A_{n}, B_{n}, \ldots, G_{2}$ in $\mathbb{R}^{n}$ equipped with a $W$-invariant euclidian form (,), $W$ the Weyl group of $R$, generated by the orthogonal reflections $\sigma_{\alpha}$ in the hyperplanes $\alpha^{*}(u)=0$, where $\alpha^{*}(u) \stackrel{\text { def }}{=}(\alpha, u)$, $u \in \mathbb{R}^{n}$. Later on, $\left\{\alpha_{1}, \ldots, \alpha_{n}\right\} \subset R$ are the simple roots for some Weyl chamber, $R_{+}$the set of positive roots $\alpha \geq 0, \sigma_{i}=\sigma_{\alpha_{i}}, u_{i}=\alpha_{i}^{*}$. Let (,) and all the $\alpha^{*}, w \in \mathbb{W}$ be extended to (bi)linear forms and automorphisms of $\mathbb{C}^{n}$. We denote by $\mathcal{O}_{n}$ the ring of analytical functions in a sufficiently small neighbourhood $U \subset \mathbb{C}^{n}$ of $u=0$.

Let $\lambda: W \rightarrow A^{*}$ be a homomorphism into the multiplicative group $A^{*}$ of some associative $\mathbb{C}$-algebra $A$. The group $W \ni w$ acts on functions $f(u)$ of $u \in \mathbb{C}^{n}$ with their values in $A$ by the formula $\lambda(w) f\left(w^{-1}(u)\right)(\lambda(w))^{-1} \stackrel{\text { def }}{=}\left({ }^{w} f\right)(u)$. For a collection $r=\left\{r_{\alpha}, \alpha \in R\right\}$ of $A$-valued functions $r_{\alpha}(u)$ and indices $1 \leq i, j \leq n$ we put

$$
D_{i}=(1 / 2) \sum_{\alpha} v_{\alpha}^{i} r_{\alpha}, F_{i j}=\left[D_{i}, D_{j}\right], v_{\alpha}^{i} \stackrel{\text { def }}{=} \partial \alpha^{*} / \partial u_{i}
$$

Let us denote by $\hat{F}_{i j}(1 \leq i, j \leq n)$ the subsum of $F_{i j}$ in which one leaves terms const $\cdot\left[r_{\alpha}, r_{\beta}\right]$ only for $\alpha, \beta \in \mathbb{Z} \alpha_{i}+\mathbb{Z} \alpha_{j}$. We assume that $r_{\alpha}$ depends only on $\alpha^{*}$ and

$$
r_{\alpha}-\left(\alpha^{*}\right)^{-1} \tau_{\alpha} \in \mathcal{O}_{U} \otimes_{C} A
$$

for some set $\left\{\tau_{\alpha} \in A \alpha \in R\right\}$.

Definition 1. A collection $r=\left\{r_{\alpha}\right\}$ is a classical $r$-matrix (of type $X_{n}$ ) iff for $1 \leq i, j \leq n$ 

a) ${ }^{w} r_{\alpha}=r_{w(\alpha)}$,
b) $r_{\alpha}+r_{-\alpha}=0$,
c) $\bar{F}_{i j} \equiv 0$.

Owing to a), there is only one or two indeterminate functions among $\left\{r_{\alpha}\right\}$, viz. $r_{\alpha_{1}}$ if $X=A, D, E$ and $r_{\alpha_{1}}, r_{\alpha_{n}}$ for other $X$ (we use here and further the numeration of the tables of [7]). As for c), there are two kinds of relations. The independent "commutativity" conditions (appearing for $n>2$ only) are as follows:

$\left[r_{\alpha_{1}}, r_{\beta}\right]=0$, where $\beta=\alpha_{3}$ in cases $X=A, D, E$ or $X_{n}=B_{3}, C_{3}, F_{4}$ and $\beta=\alpha_{3}, \alpha_{n}$ for other $B_{n}, C_{n}$. The remaining ("Yang-Baxter") equations have the form

$$
\begin{aligned}
& {\left[r_{\alpha_{1}}, r_{\alpha_{2}}\right]=\left[r_{\alpha_{1}}+r_{\alpha_{2}}, r_{\alpha_{1}}-r_{\alpha_{2}}\right]\left(X_{n} \neq B_{2}, C_{2}, G_{2}\right),} \\
& \widehat{F}_{n n-1} \equiv 0(X=B, C, G) \text { or } \widehat{F}_{23} \equiv 0 \text { for } X_{n}=F_{4} .
\end{aligned}
$$

For example, let us write down the last condition for $B, C, F$ in the important particular case when $\left[r_{\alpha}, r_{\beta}\right]=0$, if $(\alpha, \beta)=0$ (for all $\alpha, \beta \in R$ ). We obtain the relation

$$
\left[r_{\alpha}, r_{\beta}+r_{a+\beta}\right]=\left[r_{\alpha+2 \beta}, r_{\alpha+\beta}-r_{\beta}\right],
$$

where $\alpha=\alpha_{n-1}, \beta=\alpha_{n}$ for $B_{n}, \alpha=\alpha_{\bar{n}}, \beta=\alpha_{n-1}\left(C_{n}\right)$ and $\alpha=\alpha_{2}, \beta=\alpha_{3}\left(F_{4}\right)$. See [20] for some details.

In the case of $A_{n-1}$ for $A=\mathbb{E n d}\left(V^{\otimes n}\right)$, where $V$ is a $\mathbb{C}$-space, with $\lambda(w)$ operating on $V^{\otimes n}$ by the corresponding permutations of the components, one arrives at the usual classical $r$-matrices (Sklyanin, Faddeev et al.). To be more precise, the values of $r_{a_{i}} \stackrel{\text { def }}{=} r_{12}\left(u_{1}-u_{2}\right)$ should belong to $\operatorname{End}\left(V^{\otimes 2}\right)$. Then $r_{i j}=$ ${ }^{w_{r_{12}}}$ by definition, where $w(1,2, \ldots, n)=(i, j, \ldots)$, and $\left.c\right)$ has the well-known form

$$
\left[r_{12}, r_{34}\right]=0=\left[r_{12}, r_{23}\right]+\left[r_{12}, r_{13}\right]+\left[r_{13}, r_{23}\right] .
$$

Our general Definition 1 is a quasi-classical limit of the corresponding quantum one from [8]. Sklyanin connected certain $r$-matrices of type $D_{n}$ and some soliton equations with boundary conditions (see Funct. Anal. and Appl., 1987, v, 21, No. 3, p. $86-88)$.

Proposition 1. If $r$ satisfies Definition 1 then $F_{i j} \equiv 0$ for all $1 \leq i, j \leq n$ and the following W-invariant system of differential equations

$$
\partial \Phi / \partial u_{i}=\kappa D_{i} \Phi, 1 \leq i \leq n,
$$

is consistent (satisfies the corss-derivative integrability conditions) for an $A$-valued function $\Phi(u)$, any $\kappa \in C$ and $u$ from

$$
U^{*} \stackrel{\text { def }}{=}\left\{u \in C^{n}, \alpha^{*}(u) \neq 0 \text { for all } \alpha\right\} \text {. }
$$

We fix an invertible solution $\Phi^{0}$ of system (1) in a neighbourhood $U^{0} \subset U^{*}$ of some point $u^{0} \in U^{*}$. Let $\bar{u}^{0}$ be the projection of $u^{0}$ onto $\bar{U} \stackrel{\text { der }}{=} U^{*} / W, \boldsymbol{B} \stackrel{\text { def. }}{=}$ 
$\pi_{1}\left(\bar{U}, \bar{u}^{0}\right)-$ the fundamental group of $\bar{U}$. One can represent every element $\gamma \in \mathbb{B}$ by a closed path $\bar{\gamma} \ni \bar{u}^{0}$ in $\dot{U}$ and pull $\bar{\gamma}$ back to a path $\gamma^{*} \subset U^{*}$ connecting $u^{0}$ with some point $w_{\gamma} u^{0}$, where $w_{\gamma}$ is a certain element of $W$ (depending only on $\gamma$ ). We denote the analytic continuation of $\Phi^{0}$ along $\gamma^{*}$ by $\Phi_{\gamma}^{0}$ and introduce the monodromy ${ }^{66}$ matrix":

$$
T_{\gamma}=\left(\Phi^{0}\left(w_{\gamma}^{-1}(u)\right)\right)^{-1} \lambda\left(w_{\gamma}\right)^{-1} \Phi_{\gamma}^{0}(u) \in A^{*} .
$$

(One has to provide the existence of $\Phi_{\gamma}^{0}$, if $\operatorname{dim}_{\mathbf{C}} A=\infty$.) The map $\beta: \mathbb{B} \rightarrow A^{*}$, $\beta(\gamma)=T_{\gamma}$ is an anti-homomorphism: $T_{\gamma} T_{\gamma^{\prime}}=T_{\gamma^{\prime} \gamma}$ (in particular, $w_{\gamma} w_{\gamma^{\prime}}=w_{\gamma \gamma^{\prime}}$ ).

Put $s^{j}(\psi)=u^{0}+\left(\alpha_{j}, u^{0}\right)\left(\alpha_{j}, \alpha_{J}\right)^{-1}(\exp (\pi i \psi)-1) \alpha_{j}$ for $u^{0}$ from $\mathbb{R}^{n}$ with the coordinates $u_{i}^{0}>0$. The element in $\mathbb{B}$ corresponding to the projection onto $\bar{U}$ of the image of the segment $0 \leq \psi \leq 1$ with respect to $s^{J}$ will be denoted by $s_{j}$. Here and below $S_{j}=T_{s_{l}}(1 \leq j \leq n)$. The group $\mathbb{B}$ (the so-called generalized braid group - see [9]) has the system of generators $\left\{s_{i}\right\}$ with the following fundamental relations

$$
\left(s_{i} s_{j}\right)^{m / 2}=\left(s_{j} s_{i}\right)^{m / 2} \text { or } s_{i} s_{i+1} s_{i}=s_{i+1} s_{i} s_{i+1}
$$

respectively for even $m=m_{i j}\left(m_{i j}\right.$ is the order of $\left.\sigma_{i} \sigma_{j}\right)$ or for $m=3(1 \leq i<j \leq n)$.

Affime analogs. Let us transfer the previous considerations to the case of the affine Weyl group $W_{a}$ generated by the reflections $\sigma_{\alpha, k}$ in the affine hyperplanes $\alpha^{*}(u)=k \in \mathbb{Z}$. We denote the normalizer of $W_{a}$ by $\bar{W}_{a}$. Let $\bar{U}_{a}=U_{a}^{+} / \bar{W}_{a}, U_{a}^{+}=$ $\left\{u \in U, \alpha^{*}(u) \notin \mathbb{Z}\right\}$. By analogy, there should be

$$
D_{i}=(1 / 2) \sum_{\alpha \in R} v_{a}^{i} r_{a}^{a}, r_{a}^{a}=\sum_{k \in \mathbb{Z}} r_{a, k}
$$

for some set $\left\{r_{\alpha, k}, \alpha \in R, k \in \mathbb{Z}\right\}$.

Let $\lambda_{a}: \bar{W}_{a} \rightarrow A^{*}$ be a homomorphism extending $\lambda$. To describe $\lambda_{a}$ one has to know $\lambda_{a}(w)$ only for the shifts $w=\bar{\omega}_{i}: u \rightarrow u+\omega_{i}^{\prime}$, where $\left\{\omega_{i}^{\prime}\right\} \subset \mathbb{R}^{n}$ are the dual fundamental weights: $\left(\omega_{i}^{\prime}, \alpha_{j}\right)=\delta_{i j}, 1 \leq i, j \leq n$, for Kronecker's delta. These shifts $\bar{\omega}_{i}$ generate a normal subgroup $\bar{P}$ in $\bar{W}_{a}$ with the natural action of $W, \bar{W}_{a}$ is the semi-direct product $W \cdot \bar{P}$ of $W$ and $\bar{P}$.

We set formally for some $r=\left\{r_{a}, \alpha \in R\right\}$

$$
r_{\alpha_{t}}^{a}(u)=\sum_{k \in Z}\left(\lambda_{a}\left(\bar{\omega}_{i}^{k}\right) r_{\alpha_{t}}\left(u_{i}-k\right) \lambda_{a}\left(\bar{\omega}_{i}^{-k}\right)\right.
$$

under the assumption that

$$
\lambda_{a}\left(\bar{\omega}_{j}\right) r_{\alpha_{t}}=r_{\alpha_{t}} \lambda_{a}\left(\bar{\omega}_{j}\right) \text { for } i \neq j \text {. }
$$

Put $r_{\alpha}^{a}={ }^{w} r_{\alpha_{1}}$ for every $w \in W$ transforming $\alpha_{i}$ to $\alpha \in R$ ( $r_{\alpha}^{a}$ does not depend on the choice of $w$ if condition a) of Definition 1 holds true). Given a classical $r$-matrix $r$, we claim that $r^{a}=\left\{r_{\alpha}^{a}, \alpha \in R\right\}$ will be a classical $r$-matrix as well.

There are some ways to make (4) meaningful. E.g., impose the condition 


$$
\lambda_{a}\left(\bar{\omega}_{i}\right) r_{\alpha_{t}}\left(u_{i}-1\right)=r_{\alpha_{i}}\left(u_{i}\right) \lambda_{a}\left(\bar{\omega}_{i}\right), 1 \leq i \leq n,
$$

which are defining for trigonometric classical $r$-matrices. Then $r^{a}$ coincides with $r$ by definition and $\pi_{1}\left(\bar{U}_{a}, \bar{u}^{0}\right)$ for a suitable $\bar{u}^{0}$ is nothing else but the monodromy group of (1). To be more precise, one should consider (1) for $\bar{P}$-invariant $U$. Then the latter will be true.

\section{§2. The Factorization}

Let us join some of the $\left\{\alpha_{i}\right\}, 1 \leq i \leq n$ by arrows $\alpha_{i^{\prime}} \rightarrow \alpha_{i}$. One has the associated ordering: $\alpha_{i} \geqslant \alpha_{j}$ iff there exists a sequence $\alpha_{i}=\alpha_{i_{1}} \rightarrow \alpha_{i_{2}} \rightarrow \ldots \rightarrow \alpha_{i_{k}}$ $=\alpha_{j}$ or $\alpha_{i}=\alpha_{j}$. First, we assume $\left\{\alpha_{i}\right\}$ with all the arrows to form a tree $\Gamma$. The second condition is as follows. Any connected set of the Dynkin diagram for $R$ (with $\left\{\alpha_{l}\right\}$ as vertices) contains a maximal vertex relative to the above ordering. In particular, there exists $\alpha_{s}=\max _{\Gamma}\left\{\alpha_{i}\right\}, 1 \leq i \leq n$, and for every $i \neq s$ there is the only "next" vertex $\alpha_{i^{\prime}}$ connected with $\alpha_{i}$ by the arrow $\alpha_{i^{\prime}} \rightarrow \alpha_{i}$. The last arrow will be denoted by $a_{l}$.

Let $\langle i j\rangle$ be the only minimal path in the tree $\Gamma$ (not in the Dynkin diagram!) from $\alpha_{i}$ to $\alpha_{j}$. We set $\varepsilon_{k}\langle i j\rangle=0$ when $a_{k} \notin\langle i j\rangle, \varepsilon_{k}\langle i j\rangle=-1$ for $a_{k} \in\langle i i\rangle$ if $\alpha_{i}$ is closer to $\alpha_{k}$ then to $\alpha_{k^{\prime}}$; otherwise $\varepsilon_{k}\langle i j\rangle=1$. Let us denote $\max _{\Gamma}\left\{\alpha_{l}, v_{a} \stackrel{\text { def }}{=} \partial \alpha^{*} /\right.$ $\left.\partial u_{i} \neq 0\right\}$ for $\alpha \in R$ by $m_{\Gamma}(\alpha)=m(\alpha)$ and put $\mathbf{M}_{\Gamma}=\left\{m_{\Gamma}(\alpha)\right\}$. Here and elsewhere $\alpha \in R_{+}(\alpha \geq 0)$.

Changes of variables. Given $p(1 \leq p \leq n)$ we introduce the substitution $v_{\imath}=u_{i} / u_{i^{\prime}}(i \neq s), v_{s}=u_{p}$, which is called to be of type $(p, \Gamma)$ and put $o_{v}=\left(v_{i}=0\right)$ (to distinguish this point from $o=\left(u_{i}=0\right)$ ), $V^{*}=\left\{v=\left(v_{i}\right) \in V, \prod_{i=1}^{n} v_{i} \neq 0\right\}$ for some sufficiently small neighbourhood $V \in C^{n}$ of $o_{v}$. We identify points from $V^{*}$ with their preimages in $U^{*}$ and assume the image $v^{0}$ of $u^{0}$ to be in $V^{*}$.

Proposition 2. System (1) can be rewritten in terms of $v \in V^{*}$ as follows

$$
\partial \Phi / \partial v_{i}=\kappa\left(c_{i} v_{i}^{-1}+b_{i}(v)\right) \Phi, c_{i} \in A, b_{i} \in \mathcal{O}_{V} \otimes_{C} A,
$$

where $p=s$ if $\tilde{r}_{\alpha}(u) \stackrel{\text { def }}{=} \alpha^{*} r_{\alpha}(u) \neq \tau_{\alpha}$ for some $\alpha$,

$$
\begin{aligned}
c_{i}= & \sum_{\alpha} \varepsilon_{i}\langle p m\rangle \tau_{\alpha}(i \neq s), c_{s}=\sum_{\alpha} \tau_{\alpha}, \alpha \in R_{+}, \\
b_{i}(v)= & \sum_{\alpha} \tilde{r}_{\alpha}\left(\sum_{j=1}^{n} v_{\alpha}^{j} \varepsilon_{i}\langle m j\rangle \prod_{m}^{j}\right)\left(\sum_{j=1}^{n} v_{\alpha}^{j} \prod_{m}^{j}\right)^{-1} \\
& +\sum_{\alpha} \varepsilon_{i}\langle p m\rangle\left(\tilde{r}_{\alpha}(u)-\tau_{\alpha}\right)(i \neq s), b_{s}(v)=\sum_{\alpha}\left(\tilde{r}_{\alpha}(u)-\tau_{\alpha}\right),
\end{aligned}
$$

for $m=m(\alpha), \prod_{m}^{j}=\prod_{l} v_{l}$ where $a_{l} \in\langle m j\rangle, \prod_{m}^{m}=1$. 
It arises from (5) that all $c_{i}$ are pairwise commutative. It holds true for $\zeta_{m}=\sum_{m(\alpha)=m} \tau_{\alpha}, m \in M_{\Gamma}\left(\alpha \in R_{+}\right)$, since $c_{i}=\sum_{m} \varepsilon_{i}\langle p m\rangle \zeta_{m}(i \neq s), c_{s}=\sum_{m} \zeta_{m}$ and the linear span of $\left\{c_{i}\right\}$ coincides with that of $\left\{\zeta_{m}\right\}$.

Later on, $w^{\kappa} \stackrel{\text { def }}{=} \exp (\kappa z \log w)$ for any $w \in C, z \in A$ is viewed either as a formal series in $\kappa \rightarrow 0$ for arbitrary $A$ or as a holomorphic multi-valued function for a finite dimensional $A$, where $\log$ is (for the sake of definiteness) the branch with the cut off along $-i \boldsymbol{R}_{+}$. In the first case one must replace $A$ everywhere by the algebra $A[[\kappa]]$ of formal series in $\kappa$. We will assume below that the elements $\mathrm{Kad}_{c_{l}}-l$ are invertible for any $1 \leq i \leq n, l=1,2, \ldots$, where $a d_{c}: x \rightarrow[c, x]$ is a linear operator on $A \ni x$.

Proposition 3. There exists for any $f \in A$ commuting with each $\zeta_{m}$ the unique solution of (5) in the form of

$$
\Phi(v)=\left(f+\Phi^{\prime}\right) \prod_{m \in M_{\Gamma}} u_{m}^{\kappa_{m}}
$$

where $\prod_{m} u_{m}^{\kappa \zeta_{m}}=\prod_{i=1}^{n} v_{i}^{\kappa c_{i}}, v$ belongs to a sufficiently small $V^{*}, \Phi^{\prime} \in A \otimes_{C} \mathcal{O}_{V}$, $\Phi^{\prime}\left(o_{v}\right)=0$ (see e.g. [10]).

Let us now put $p=s$ and take the solution (6) for $f=1$ as $\Phi^{0}(v)$ (or $\Phi^{0}(u)$ ). It depends on $\left\{\alpha_{i}\right\}$ (or the corresponding Weyl chamber $C$ ) and on $\Gamma$ (to be more precise on the restriction of the ordering $\geqslant$ onto $M_{\Gamma}$ ). It does not depend on the choice of a concrete $v^{0}$. We will write down $\Phi^{0}=\Phi^{0}(u ; C, \Gamma)$. It is evident that ${ }^{w} \Phi^{0}=\Phi^{0}(u ; w(C), \Gamma)$ for $w \in W$. Given two pairs $(C, \Gamma)$ and $(\widetilde{\mathcal{C}}, \widetilde{\Gamma})$, some points $u^{0}, \tilde{u}^{0}$ (with their images in $V, \widetilde{V}$ ) and a path $\gamma^{*} \subset U^{*}$ joining $u^{0}$ and $\tilde{u}^{0}$, denote by $\Phi_{\gamma}^{0}$ the analytic continuation of $\Phi^{0}$ along $\gamma^{*}$. Here $\gamma$ is the homotopy class of $\gamma^{*}$ among all the paths in $U^{*}$ from $V$ to $\widetilde{V}$. Suppose $I=I_{\gamma} \subset(1, \ldots, n)$ to be a set such that for any $\varepsilon>0$ there exists $\gamma^{*} \in \gamma$ satisfying the inequalities

$$
\left|v_{i}\left(\gamma^{*}\right)\right|<\varepsilon \text { for all } i \in \mathbb{I} \text {. }
$$

Let $\widetilde{\Phi}^{0}$ be the solution of Proposition 3 for another pair $(\widetilde{C}, \widetilde{\Gamma})$. The proof of the following theorem is based on analysis of the expansions of (6) in a neighbourhood of $\left\{v, v_{j}=0\right.$ for $\left.j \notin \mathbb{I}\right\}$. These statements generalize the factorization principle by Tsuchiya, Kanie. But we use a direct consideration of (1) instead of the operator formalism from [2].

Theorem 1. 1) The "transfer matrix" $\mathscr{T}=\mathscr{T}(C, \Gamma ; \widetilde{C}, \widetilde{\Gamma})=\left(\widetilde{\Phi}^{0}\right)^{-1} \Phi_{\gamma}^{0} \in A^{*}$ does not depend on $u, v$ and can be calculated as follows. Reduce (5) to some system $\left(5^{\prime}\right)$ by throwing away all the $b_{i}$ for $i \notin I$ and replacing $b_{i}(i \in I)$ by the function $b_{i}^{\prime}$ obtained from $b_{i}$ after the substitution $v_{j}=0$ for $j \notin I$. Then the counterpart $\mathscr{T}^{\prime}$ of $\mathcal{T}$ for $\left(5^{\prime}\right)$ coincides with $\mathcal{T}$. 
2) Each rational function ${ }^{w^{\prime}} v_{i}, w^{\prime}=w^{-1}$, is regular at $o_{v}$ or has a pole at this point, i.e. the value $v_{i}\left(w\left(o_{v}\right)\right)$ is well-defined (belongs to $C \cup \infty$ ) for $1 \leq i \leq n$, $w \in W$. One can find $\gamma$ for $\tilde{u}^{0}=w u^{0}$ with the above property for $I=I(w)=$ $\left\{i, v_{i}\left(w\left(o_{v}\right)\right) \neq 0\right\}$. Then $T_{\gamma}$ for any such $\gamma$ (see (2)) exactly coincides with its analog $T_{\gamma}^{\prime}$ for $\left(5^{\prime}\right)$ with $I=I_{\gamma}=I(w)$ defined by (2) where we insert $v \rightarrow w\left(o_{v}\right)$.

A particular case. We use the notations from the tables of [7]. Let $s=1$ and $i^{\prime} \stackrel{\text { def }}{=} i-1$ except the cases $n^{\prime}=n-2$ for $X_{n}=D_{n}$ and $2^{\prime}=4,3^{\prime}=1$ for $E$. We apply the substitution of type $(p=1)$. Then $I\left(\sigma_{k}\right)=\left\{j, j^{\prime} \neq k\right\}$. This set is empty for $k=n$ and also for $k=n-1$ in case $X_{n}=D_{n}$. It consists of two elements for $k=n-2, X_{n}=D_{n}$ and for $k=4, X=E$. Elsewise $I\left(\sigma_{k}\right)=\{k+1\}$. To calculate $S_{k}=T_{s_{k}}$ one can use $\left(5^{\prime}\right)$, where $b_{j}^{\prime} \equiv 0$ for $j^{\prime} \neq k$ and

$$
b_{j}^{\prime}=\sum_{\alpha} v_{\alpha}^{j}\left(v_{\alpha}^{k}+\sum_{i^{\prime}=k} v_{\alpha}^{i} v_{i}\right)^{-1} \tau_{\alpha},
$$

where $j^{\prime}=k$ and $\alpha$ runs through all the positive roots with $v_{\alpha}^{i} \neq 0$ which are some linear combinations only of $\alpha_{l}$ with $l \geq k$ (and also $\alpha_{2}$ for $X=E, k=4$ ). We see that $\left(5^{\prime}\right)$ contains only $\left\{\tau_{\alpha}\right\}$.

Corollary 1. 1) The monodromy matrices $\{T, S\}$ and transfer matrices $\{\mathscr{T}\}$ for any chambers $C$ and trees $\Gamma$ depend only on $\left\{\tau_{\alpha}\right\}$ (not on the whole r-matrix).

2) Every element $S_{k}$ for $1 \leq k \leq n$ is conjugated in $A^{*}$ with $\tau_{\alpha_{h}} \exp \left(\pi i \kappa \tau_{\alpha_{k}}\right)$.

To prove 2) one can use the substitution $(p, \Gamma)$ if $p=s=\max _{\Gamma}$ and $\alpha_{k}$ is a minimal vertex (such a $\Gamma$ exists for each $k$ ). In this case $I\left(\sigma_{k}\right)=\varnothing$ and $c_{k}=\tau_{\alpha_{k}}$.

\section{§3. Basic Examples}

First we consider the group algebra $C[W]=\bigoplus_{w \in W} C w$ with the natural inclusion $W \rightarrow C[W]$ as the pair $(A, \lambda)$.

Theorem 2. 1) The collection $r_{\alpha}=\chi_{l}\left(\alpha^{*}\right)^{-1} \sigma_{\alpha}$ for $\alpha \in R$, where $l=l(\alpha)$ is 1 for long and 0 for short roots $\left(\chi_{0,1} \in C\right)$, is a classical A-valued r-matrix.

2) The mapping $\mathbb{B} \ni \gamma \rightarrow T_{\gamma} \in A^{*}$ for sufficiently general $q_{l}=\exp \left(\pi i \kappa \chi_{l}\right)$, $l=0,1$, induces an isomorphism of $A$ and the quotient algebra $\boldsymbol{H}_{w}=\boldsymbol{H}_{w}^{4}$ o.1 of $\mathbb{C}[\boldsymbol{B}]$ by the relations $\left(s_{k}-q_{l}\right)\left(s_{k}+q_{l}^{-1}\right)=0$, which are imposed for $1 \leq k \leq n$ $\left(l=l\left(\alpha_{k}\right)\right)$.

Statement 1) results from the construction of quantum $R$-matrices for $A$ from [8]. It could be interesting to compare statement 2) with the Benson-Curtis theorem and Lusztig isomorphisms between $C[W]$ and Hecke algebras.

For $W=S_{n}$ (in case $A_{n-1}$ ) one can follow [3,4] and generalize assertion 1). We use the notations from [7]: $\alpha_{i}=\varepsilon_{i}-\varepsilon_{i+1} 1 \leq i<n, \alpha=\varepsilon_{i}-\varepsilon_{j}(i<j)$, where $S_{n}$ acts on $\varepsilon=\left(\varepsilon_{i}\right)$ by permutations, $\sigma_{i}=(i i+1),(i j)$ are the transpositions. Let 
$A$ (equipped with a homomorphism $\lambda: \mathbb{S}_{n} \rightarrow A^{*}$ ) contain elements $\tau_{\alpha}=\tau_{i j}=$ $\tau_{j l}(1 \leq i<j \leq n)$ and $x_{i}(1 \leq i \leq n)$ with the relations

$$
\begin{gathered}
{\left[\tau_{i j}, \tau_{i k}+\tau_{j k}\right]=0=\left[\tau_{i j}, \tau_{k l}\right],{ }^{w} \tau_{i j}=\tau_{w(i) w(J)},} \\
{\left[x_{i}, x_{j}+\tau_{i j}\right]=0=\left[\tau_{i j}, x_{i}+x_{j}\right],{ }^{w} x_{i}=x_{w(i)}}
\end{gathered}
$$

for pairwise distinct $i, j, k, l, w \in \mathbb{S}_{n}$. Then for $1 \leq i \leq n$ the system

$$
\partial \Phi / \partial z_{i}=\kappa\left(x_{i}\left(z_{i}-z_{n+1}\right)^{-1}+\sum_{j \neq i} \tau_{i j}\left(z_{i}-z_{j}\right)^{-1}\right) \Phi
$$

is consistent for any constant $z_{n+1} \in \mathbb{C}$ (here the $S_{n}$-symmetries of $\tau, x$ are not significant). System (8) is $\mathbb{S}_{n}$-invariant under the action of $\mathbb{S}_{n}$ on $\left\{z_{1}, \ldots, z_{n}\right\}$ (dual to $\left\{\varepsilon_{1}, \ldots, \varepsilon_{n}\right\}$ ) by permutations together with action (7) on $\left\{\tau_{i j}, x_{i}\right\}$.

Later on, $\tau_{i j}=(i j)$ and $A$ is generated by $C\left[S_{n}\right]$ and $\left\{x_{i}, 1 \leq i \leq n\right\}$ with the defining relations $(7 b)$. This algebra is isomorphic to the degenerate affine Hecke algebra $\mathcal{H}_{n}^{\prime}$. The latter is defined (Drinfeld) by adjoining to $\mathbb{C}\left[\mathbb{S}_{n}\right]$ pairwise commutative independent $y_{1}, \ldots, y_{n}$ with the relations

$$
\begin{gathered}
\sigma_{i} y_{i+1}-y_{i} \sigma_{i}=1=y_{i+1} \sigma_{i}-\sigma_{i} y_{i}, 1 \leq i<n, \\
{\left[\sigma_{i}, y_{j}\right]=0 \text { for } j \neq i, i+1,1 \leq j \leq n .}
\end{gathered}
$$

To identify $A$ and $\mathcal{H}_{n}^{\prime}$ one has to put

$$
x_{i}=-y_{i}-\sum_{n \geq j>i}(i j), 1 \leq i \leq n .
$$

We will give a version of (8) for $W$ of other classical types. Let us construct the group $\widetilde{S}_{n}$ (the Weyl group of type $B_{n}, C_{n}$ ) by adjoining to $S_{n}$ pairwise commuting elements $\rho_{1}, \ldots, \rho_{n}$ with the relations $w \rho_{i} w^{-1}=\rho_{w(i)}, \rho_{i}^{2}=1$, $1 \leq i \leq n, w \in \mathbb{S}_{n}$, acting on $\mathbb{C}^{n} \ni z=\left(z_{i}\right)$ by the formulas $\rho_{i}\left(z_{j}\right)=(-1)^{\delta_{1 /}} z_{J}$ $\left(1 \leq i, j \leq n, \delta_{i j}-\right.$ is the Kronecker symbol).

We can add these $\left\{\rho_{i}\right\}$ to $\mathscr{H}_{n}^{\prime}$ with the extra relations $\left[\rho_{i}, x_{j}\right]=0$ for $i \neq j$. The resulting algebra will be denoted by $\hat{\mathscr{H}}_{n}^{\prime}$. Its subalgebra $\widetilde{\mathscr{H}}_{n}^{\prime}$ generated by $C\left[\widetilde{\mathbf{S}}_{n}\right]$ and

$$
\tilde{x}_{i}=\left(\chi \rho_{i}+x_{i}+\rho_{i} x_{i} \rho_{i}\right) / 2,1 \leq i \leq n,
$$

for $\chi \in C$ will be called the degenerate affine algebra of type $\mathbb{B}_{n}$ or $\mathbb{C}_{n}$ and briefly denoted by $\tilde{A}$. We will use the notations

$$
(\widetilde{i j})=\rho_{i} \rho_{j}(i j), \beta_{i j}=\left(\rho_{i} \rho_{j}+1\right) / 2, \tilde{y}_{i}=-\tilde{x}_{i}-\sum_{n \geq j>i} \beta_{i j}(i j) .
$$

The algebra $\tilde{A}$ can be defined over $C\left[\widetilde{\boldsymbol{S}}_{n}\right]$ in an abstract way by the relations

$$
\sigma_{i} \tilde{y}_{i+1}-\tilde{y}_{i} \sigma_{i}=\beta_{i i+1}=\tilde{y}_{i+1} \sigma_{i}-\sigma_{i} \tilde{y}_{i},\left[\tilde{y}_{i}, \tilde{y}_{k}\right]=0,
$$




$$
\left[\sigma_{i}, \tilde{y}_{k}\right]=\left[\rho_{k}, \tilde{y}_{j}\right]=0 \text { for } 1 \leq j, k \leq n, k \neq i, i+1 .
$$

We note that one has to consider in case $D_{n}$ the subalgebra of $\widehat{\mathscr{H}}_{n}^{\prime}$ generated by $S_{n}, \rho_{i} \rho_{j}$ and $x_{i}+\rho_{i} x_{i} \rho_{i}(1 \leq i, j \leq n)$. The terms $\chi \rho_{i}$ in the formulas for $\tilde{x}_{i}$ (and $\tilde{y}_{i}$ ) do not alter relations (9) and will be convenient only in the next sections.

Assertion 1) of Theorem 2 (for $B_{n}, C_{n}$ ) can be rewritten in terms of $\left\{z_{i}\right\}$ (dual to $\left\{\varepsilon_{i}\right\}$ from the corresponding tables of [7]) and also generalized by adding $\left\{x_{i}\right\} \subset \widehat{\mathscr{H}}_{n}^{\prime}$ as follows. We claim that the system $(1 \leq i \leq n)$

$$
\begin{aligned}
\partial \Phi / \partial z_{i}= & \kappa\left(\sum_{\jmath \neq i}(i j)\left(z_{i}-z_{j}\right)^{-1}+(\widetilde{i j})\left(z_{i}+z_{j}\right)^{-1}\right) \Phi \\
& +\kappa\left(\chi \rho_{i} z_{i}^{-1}+x_{i}\left(z_{i}-z_{n+1}\right)^{-1}+\rho_{i} x_{i} \rho_{i}\left(z_{i}+z_{n+1}\right)^{-1}\right) \Phi
\end{aligned}
$$

for $z_{n+1} \in C$ (an arbitrary constant) and an $\widehat{\mathscr{H}}_{n}^{\prime}$-valued function $\Phi(z)$ is consistent and $\widetilde{S}_{n}$-invariant. We will study (10) later only for $z_{n+1}=0$ and after the substitution

$$
v_{k}=\left(z_{k}-z_{k+1}\right)\left(z_{k-1}-z_{k}\right)^{-1}, 1<k<n, v_{1}=z_{1}-z_{2}, v_{n}=z_{n}\left(z_{n-1}-z_{n}\right)^{-1}
$$

(cf. the particular case after Theorem 1 ). We arrive at the consistent $\widetilde{A}$-valued system

$$
\begin{aligned}
\partial \Phi / \partial v_{1} & =\kappa \tilde{c}_{1} v_{1}^{-1} \Phi, \kappa^{-1}\left(\partial \Phi / \partial v_{k}\right) \Phi^{-1} v_{k}-\tilde{c}_{k} \\
& =2 \sum_{i<k} V_{i k}^{n+1} \tilde{x}_{i}+\sum_{j>k>1} V_{i k}^{j}(i j)+\sum_{j>i<k} \widetilde{V}_{i k}^{j}(\widetilde{i j})
\end{aligned}
$$

for $\tilde{c}_{k}=-2 \sum_{i \geq k} \tilde{y}_{i}, V_{i k}^{j}=\left(\sum_{l=k}^{j-1} \prod_{i}^{l}\right)\left(1+\sum_{l=i+1}^{j-1} \Pi_{i}^{l}\right)^{-1}$,

$$
\tilde{V}_{i k}^{j}=\left(\sum_{l=k}^{j-1} \Pi_{i}^{l}+2 \sum_{l=j}^{n} \Pi_{i}^{j}\right)\left(1+\sum_{l=i+1}^{j-1} \Pi_{i}^{l}+2 \sum_{l=j}^{n} \Pi_{i}^{l}\right)^{-1}
$$

where $\prod_{i}^{l}=\prod_{m=i+1}^{l} v_{m}, 1<k \leq n$.

Further we will consider system (8) only for $\tau_{i j}=(i j)$ and $z_{n+1}=0$ as well. After the same substitution (11) we arrive at the system which can be obtained from (12) if one puts $\rho_{i} \equiv 0$ and $(\widetilde{i j}) \equiv 0$ for every $i, j$. We will use this remark to calcualte monodromy matrices for $A_{n-1}$ and $B_{n}$ simultaneously. Let us denote (12) without $\rho_{i}$ and $(\widetilde{i j})$ by $(12 \mathrm{~A})$. One has $\tilde{x}_{i}=x_{i} / 2, \tilde{y}_{i}=y_{i} / 2$ in $(12 \mathrm{~A})$.

\section{§4. The Monodromy}

We choose the initial point $z^{0}=\left(z_{i}^{0}\right) \in \boldsymbol{R}^{n}$ with its image $v^{0}=\left(v_{i}^{0}\right)$ in the domain $\left\{0>v_{i}^{0}>-1 / 2\right\}$ for (12) and in $\left\{0<v_{i}^{0}<1 / 2\right\}$ for $(12 \mathrm{~A})$. Let $\Phi^{0}$ be the solution of type (6) for (12) or $(12 \mathrm{~A})$ and $f=1$. Our aim is to caluclate the 
monodromy matrices $\widetilde{S}_{k}$ in case (12) and $S_{k}$ for (12A) which correspond to the paths

$$
\begin{aligned}
& \tilde{s}^{k}(\psi)=\left(z_{j}^{0}+\left(\delta_{k j}+\delta_{k+1 j}\right)(\exp (\pi i \psi)-1)\left(z_{k}^{0}+z_{k+1}^{0}\right) / 2\right), \\
& s^{k}(\psi)=\left(z_{j}^{0}+\left(\delta_{k j}-\delta_{k+1 j}\right)(\exp (\pi i \psi)-1)\left(z_{k}^{0}-z_{k+1}^{0}\right) / 2\right),
\end{aligned}
$$

where $\psi$ runs along the segment [01], $1 \leq k<n, 1 \leq j \leq n$ and matrices $\widetilde{R}_{l}, R_{l}$ respectively for (12), (12A) and the paths

$$
\tilde{r}^{l}=\left(z_{j}^{0} \exp \left(\pi i \psi \delta_{j l}\right), r^{l}=\left(z_{j}^{0} \exp \left(2 \pi i \psi \delta_{j l}\right)\right), 1 \leq l \leq n .\right.
$$

Here we follow Section 1 with some natural modifications which will be explained now.

Equations (8) for $z_{n+1}=0$ (and their transformations (12A)) are well defined in the domain $Z=\left\{z \in \mathbb{C}^{n}, \prod_{i \neq j}\left(z_{i}-z_{j}\right) z_{i} \neq 0\right\}$ and give us the monodromy homomorphism from $\mathbb{B}=\pi_{1}\left(\bar{Z}, \bar{z}^{0}\right)$ to $A^{*}$ for $\bar{Z} \stackrel{\text { def }}{=} Z / S_{n}$. The paths $\left\{s^{k}\right\}$ are the natural pullbacks to $Z$ of the $\left\{s_{k}\right\}$ from Section 1 in case $A_{n-1}$ (we use the notations of the tables from [7]). Their images $\left\{s_{k}\right\}$ in $\mathbb{B}$ satisfy the same relations (3). The image $r_{n}$ of $r^{n}$ in $B$ satisfies the relation $s_{n-1} r_{n} s_{n-1} r_{n}=$ $r_{n} s_{n-1} r_{n} s_{n-1}$. These relations together are fundamental for $\mathbb{B}$, generated by $\left\{s_{1}, \ldots, s_{n-1}, r_{n}\right\}$.

The corresponding group $\mathbb{B}$ for (12) is $\pi_{1}\left(\bar{Z}^{\prime}, \bar{z}^{0}\right)$, where $Z^{\prime}=\{z \in Z$, $\left.\prod_{i, j}\left(z_{i} \pm z_{j}\right) \neq 0\right\}, \bar{Z} \stackrel{\text { def }}{=} Z^{\prime} / \widetilde{S}_{n}$. In fact, this group was introduced in Section 1 for $X_{n}=B_{n}$. The images $\left\{\tilde{s}_{1}, \ldots, \tilde{s}_{n-1}, \tilde{r}_{n}\right\}$ of $\left\{\tilde{s}^{k}, \tilde{r}^{n}\right\}$ in $\widetilde{B}$ are absolutely analogous to $s_{1}, \ldots, s_{n-1}, r_{n}$, but for another $u^{0}$. They generate $\widetilde{B}$ and satisfy (3) for $\tilde{s}_{n}=\tilde{r}_{n}$ as well. Notice that the mapping $s_{k} \rightarrow \tilde{s}_{k}, r_{n} \rightarrow \tilde{r}_{n}$ induces an isomorphism $\mathbb{B} \rightarrow \widetilde{\mathbb{B}}$.

The algebras $A, \widetilde{A}$ are infinite dimensional. Therefore we are to be precise when dealing with monodromy matrices. Further all the expressions and formulas make sense and are valid in any finite dimensional representations of $A, \widetilde{A}$ or as formal series in $\kappa \rightarrow 0$. In particular, one can write down that $S_{k}, R_{l} \in A^{*}, \widetilde{S}_{k}$, $\widetilde{R}_{k} \in \widetilde{A}^{*}$ only by some abuse of notations.

We remind the definition of the affine Hecke algebra $\mathscr{H}_{n}^{q}$ (for $G L_{n}$ ) in the Bernstein, Zelevinsky form (see [11]). It is the quotient algebra of the group algebra $C[B]$ by the relations

$$
\left(s_{k}-q\right)\left(s_{k}+q^{-1}\right)=0,1 \leq k<n, q \in \mathbb{C}^{*} .
$$

The letters $T_{1}, \ldots, T_{n-1}$ are in common use for the images of $\left\{s_{k}\right\}$ in $\mathscr{H}_{n}^{q}$. The image of $r_{n}^{-1}$ will be denoted by $Y_{n}$. Put $Y_{l}=T_{l}^{-1} \ldots T_{n-1}^{-1} Y_{n} T_{n-1} \ldots T_{l}^{-1}$. It results from the relations in $B$ that all $\left\{Y_{l}\right\}$ are pairwise commutative. Now we are in a position to formulate the main 
Theorem 3. 1) Matrices $\left\{\widetilde{S}_{k}\right\}$ satisfy relations (13) and the mapping $\tilde{s}_{k} \rightarrow$ $\widetilde{S}_{k}, \tilde{r}_{n} \rightarrow \widetilde{R}_{n}$ induces for generic $q=\exp (\pi i \kappa)$ an isomorphism $\widetilde{\beta}: \mathscr{H}_{n}^{q} \rightarrow \tilde{A}$ (defined in the category of finite dimensional representations or as formal series in $\kappa$ ).

2) The following relations are true for generic $\kappa$ :

$$
\begin{gathered}
\left(\widetilde{S}_{k}+\left(q-q^{-1}\right)\left(q^{2\left(\tilde{y}_{h}^{\prime}-\tilde{y}_{k+1}^{\prime}\right)}-1\right)^{-1}\right) g^{\left(\tilde{y}_{h}^{\prime}-\tilde{y}_{k+1}^{\prime}\right)} \\
=\sigma_{k}+\beta_{k k+1}\left(\tilde{y}_{k}-\tilde{y}_{k+1}\right)^{-1}, \widetilde{R}_{l}^{-1}=q^{2 \tilde{y}_{l}}=\exp \left(2 \pi i \kappa \tilde{y}_{l}\right), 1 \leq l \leq n, 1 \leq k<n,
\end{gathered}
$$

where $g(y)=\exp (2 \pi i \kappa y) \Gamma(\kappa(y-1)) \Gamma(\kappa(y+1)) \Gamma^{-2}(\kappa y)\left(y^{2}-1\right) y^{-2}, \Gamma$ is the gamma function, $\tilde{y}_{k}^{+} \stackrel{\text { def }}{=} \tilde{y}_{k}-\left(\rho_{k}-1\right) /(4 \kappa), \tilde{y}_{k}$ are from $(9)$.

3) All these assertions hold good for $A,\left\{R_{l}, S_{k}\right\}$ instead of $\widetilde{A},\left\{\widetilde{R}_{l}, \widetilde{S}_{k}\right\}$ after the substitution $\tilde{y}_{l}^{+}=\tilde{y}_{l}=y_{l}, \beta_{k+1}=1$, where $1 \leq l \leq n>k \geq 1$.

We can express $\left\{\widetilde{S}_{k}, S_{k}\right\}$ explicitly by formulas (14) in terms of $\left\{\sigma_{k}, \rho_{k} \rho_{k+1}\right.$, $\left.\tilde{y}_{k}^{+}-\tilde{y}_{k+1}^{+}\right\}$regarding $\Gamma$ as some integral or reducing it to the scalar $\Gamma$-function after a diagonalization of $\tilde{y}_{k}^{+}-\tilde{y}_{k+1}^{+}$(if the latter is possible). Notice that one can verify directly that the resulting formulas for $\left\{\widetilde{S}_{k}\right\}$ or $\left\{S_{k}\right\}$ induce some isomorphisms $\tilde{A} \underset{\rightarrow}{\rightarrow} \mathscr{H}_{n}^{q} \leftleftarrows A$ (independently of the origin of $\left\{\widetilde{S}_{k}, S_{k}\right\}$ ). By the way, it gives us certain simple conditions for $\kappa$ ensuring the existence of these isomorphisms. There are some straightforward generalizations of statement 2) for other Hecke algebras (without any monodromy technique)*.

Corollary 2. Setting $x_{j} \equiv 0$ for all $1 \leq j \leq n$ in formulas (14) the resulting expressions for $\left\{S_{k}, \widetilde{S}_{k}\right\}$ in terms of

$$
y_{i}=-\sum_{j>i}(i j), \quad \tilde{y}_{i}^{*}=-\sum_{j>i} \beta_{i j}(i j)-\left(\left(\rho_{i}-1\right)(2 \kappa)^{-1}+\chi \rho_{i}\right) / 2
$$

give one isomorphisms between $\mathbb{C}\left[S_{n}\right], C\left[\widetilde{S}_{n}\right]$ and the corresponding Hecke algebras $H_{w}$ of type $A_{n-1}$ or $B_{n}$ respectively (cf. Theorem 2).

\section{§5. Using Young Bases}

To prove Theorem 3 it is sufficient to check formulas (14) in every representation $M_{u, \varepsilon}$ of $\widetilde{A}$ or $A$ defined for arbitrary sets $u=\left(u_{1}, \ldots, u_{n}\right) \in C^{n}, \varepsilon=\left(\varepsilon_{1}, \ldots\right.$, $\left.\varepsilon_{n}\right) \in Z_{2}^{n}$ as follows. Let $M_{u, \varepsilon}$ be $C\left[S_{n}\right]$ with the left regular action of $S_{n}$ as a $S_{n^{-}}$ module. We introduce the action of $\left\{\tilde{y}_{j}, \rho_{j}\right\}$ by the relations

$$
\tilde{y}_{j}(1)=u_{j}, \rho_{j}(1)=\varepsilon_{j} \in\{ \pm 1\}=Z_{2}, 1 \leq j \leq n .
$$

\footnotetext{
* It was proved that some analogs of (14) give us isomorphisms of affine Hecke algebras and their graded (degenerate) versions in the recent paper by G. Lusztig (J. of AMS, 1989, v. 2, No. 3, pp. 599-635).
} 
In fact one should prove (14) only for $\left\{u_{i}\right\}$ being in a general position (and then use the deformation procedure). Further we will apply and generalize to $\widetilde{\mathscr{H}}_{n}^{\prime}$ some results by Zelevinsky, Rogawski (see $[12,13])$ and those from $[14-16]$ on the representation theory of $\mathscr{H}_{n}^{q}, \mathscr{H}_{n}^{\prime}$.

It is possible to define the collection $\left\{f_{s}(u, \varepsilon), s \in \mathbb{S}_{n}\right\}$ of functions in $u \in \mathbb{C}^{n}$, $\varepsilon \in \mathbb{Z}_{2}^{n}$ taking values in $\mathbb{C}\left[\mathbb{S}_{n}\right]$ by the relations

$$
\begin{aligned}
& f_{s_{k}}(u, \varepsilon)=\sigma_{k}+\left(u_{k}-u_{k+1}\right)^{-1}\left(\varepsilon_{k} \varepsilon_{k+1}+1\right) / 2,1 \leq k<n, \\
& f_{s t}(u, \varepsilon)=f_{s}(t(u), t(\varepsilon)) f_{t}(u, \varepsilon) \text { for } s t>t, s, t \in \mathbb{S}_{n} .
\end{aligned}
$$

Here $s t>t$ iff the length $l(s t)$ of a reduced decomposition for $s t$ in $S_{n}$ is equal to $l(\mathrm{~s})+l(t), \mathbb{S}_{n}$ acts on $u, \varepsilon$ by permutations.

A generalized skew Young diagram (cf. [14]) is a collection

$$
\mu=\left\{\mu^{ \pm r}, d( \pm r) \in C, 1 \leq r \leq n\right\}, \text { where }
$$

$d(r)-d\left(r^{\prime}\right) \notin \mathbb{Z} \boxplus d(-r)-d\left(-r^{\prime}\right)$ for $r \neq r^{\prime}, \mu^{\xi r}$ for $\xi= \pm 1,1 \leq r \leq n$ is a usual skew Young diagram $\mu^{\xi r}=\left\{m_{i}^{\xi} \geq \widetilde{m}_{i}^{\xi r}\right\} \subset \mathbb{Z}(1 \leq i \leq n)$ satisfying by definition the conditions $\widetilde{m}_{\Gamma}^{\xi r}=0, m_{1}^{\xi r} \geq \ldots \geq m_{n}^{\xi r}, \widetilde{m}_{1}^{\xi r} \geq \ldots \geq \widetilde{m}_{n}^{\xi r}$. We assume that $\sum_{i, \xi, r}\left(m_{i}^{\xi r}-\widetilde{m}_{i}^{\xi r}\right)=n$. We can number the set $\left\{(\xi, r, i, j), \widetilde{m}_{1}^{\xi r}<j \leq m_{i}^{\xi r}\right\}$ by (lower) indices $k(1 \leq k \leq n)$ in the "natural" order:

$$
\begin{gathered}
\{k>m\} \text { if }\left\{\xi_{k}<\xi_{m}\right\} \text { or if }\left\{\xi_{k}-\xi_{m}=0<r_{k}-r_{m}\right\} \text { or } \\
\left\{\xi_{k}-\xi_{m}=r_{k}-r_{m}=0<i_{k}-i_{m}\right\} \text { or }\left\{\xi_{k}-\xi_{m}=r_{k}-r_{m}=i_{k}-i_{m}=0<j_{k}-j_{m}\right\} .
\end{gathered}
$$

Let us introduce the permutation $\omega=\omega_{\mu} \in S_{n}$ of the indices $\{k\}$ which does not change $\xi_{k}, r_{k}$ and has the defining property:

$$
\text { either }\left\{j_{\omega(k)}<j_{\omega(m)}\right\} \text { or }\left\{j_{\omega(k)}-j_{\omega(m)}=0>i_{\omega(k)}-i_{\omega(m)}\right\}
$$

for any $k>m\left(\xi_{k}=\xi_{m}, r_{k}=r_{m}\right)$. Put

$$
\begin{aligned}
& u_{k}^{0}=i_{k}-j_{k}+d\left(\xi_{k} r_{k}\right)-\chi \xi_{k} / 2-\left(\xi_{k}-1\right)(4 \kappa)^{-1}, 1 \leq k \leq n, \\
& \hat{u}_{k}^{0}=u_{k}^{0}+\gamma\left(i_{k}-1\right), \gamma \in \mathbb{C}, \hat{u}^{0} \stackrel{\text { def }}{=} \hat{u}_{\mu}^{0}=\left(\hat{u}_{k}^{0}, 1 \leq k \leq n\right), \varepsilon_{k}^{0}=\xi_{k} .
\end{aligned}
$$

We note that the below construction of bases for $\widetilde{\mathscr{H}}_{n}^{\prime}$ will not depend on $\chi, \kappa$ on $d( \pm 1)$ and on $d(\xi r)$ for "empty" $\mu^{\xi r}$. Moreover, the representations from the next proposition modulo isomorphisms do not depend on the order of pairs in the set $\left\{\left(\mu^{+r}, d(+r)\right)\right\}$ or in $\left\{\left(\mu^{-r}, d(-r)\right)\right\}$. The terms with $\chi, \kappa$ in the formulas for $u^{0}$ and the difference $d(+1)-d(-1)$ will be significant only later for $\mathcal{H}_{n}^{q}$.

Proposition 4. Any irreducible $\widetilde{\mathscr{H}}_{n}^{\prime}$-module with the semi-simple action of $\left\{\tilde{y}_{1}, \ldots, \tilde{y}_{n}\right\}$ is isomorphic to the $\widetilde{\mathscr{H}}_{n^{\prime}}^{\prime}$ submodule $M^{\mu}=\bigoplus_{s \geq \omega} \mathbb{C} f_{s}^{0} \subset M_{u^{0}, \varepsilon^{0}}$ for some $\mu$, where $f_{s}^{0}=\lim _{\gamma \rightarrow 0} f_{s}\left(\hat{u}^{0}, \varepsilon^{0}\right) \in \mathbb{C}\left[\mathbb{S}_{n}\right]$. The converse (for any $\left.\mu\right)$ is true as well. One 
has for $s \geq \omega$

$$
\rho_{j}\left(f_{s}^{0}\right)=\varepsilon_{j^{\prime}}^{0} f_{s}^{0}, \tilde{y}_{j^{\prime}}^{\prime}\left(f_{s}^{0}\right)=u_{j}^{0} f_{s}^{0}, j^{\prime} \stackrel{\text { def }}{=} s^{-1}(j) .
$$

We note that a general $M_{u, \varepsilon}$ can be represented as $M^{\mu}$ for an appropriate set $\{d( \pm r)\}$ if $\omega=i d$ (e.g., each $\mu^{\xi r}$ contains no more than one box). We need only these $M_{u, \varepsilon}$ to prove the theorem.

It is possible to generalize the main idea of this construction for any group $\widetilde{\boldsymbol{S}}_{n}=\boldsymbol{S}_{n} P$ if one can find in $C[P]$ elements $\pi_{i j}=\pi_{j i}(1 \leq i<j \leq n)$ with the properties

$$
s \pi_{i j} s^{-1}=\pi_{s(i) s(j)},(i j) p(i j) \pi_{i j}=\pi_{i j} p
$$

for any $i<j, s \in S_{n}, p \in P$. E.g. $\pi_{i j}=\beta_{i j}$ for $\widetilde{\boldsymbol{S}}_{n}$ above. Without going into detail we must define an analog of $\widetilde{\mathscr{H}}_{n}^{\prime}$ by relations (9) with $\pi_{i i+1}$ in place of $\beta_{i+1}$ and use $f_{s_{k}}(u)=\sigma_{k}+\left(u_{k}-u_{k+1}\right)^{-1} \pi_{k k+1}$ instead of $f_{s_{k}}(u, \varepsilon)$.

Calculation of $\left\{\boldsymbol{S}_{\boldsymbol{k}}, \widetilde{\boldsymbol{S}}_{\boldsymbol{k}}\right\}$ in $\boldsymbol{M}^{\mu}$. Theorem 1 shows that when calculating $\widetilde{\boldsymbol{S}}_{k-1}$, $S_{k-1}$ one has to solve the $\operatorname{End}_{C}\left(M^{\mu}\right)$-valued system

$$
\begin{aligned}
& \kappa^{-1}\left(\partial \Phi / \partial v_{j}\right) \Phi^{-1}=\tilde{c}_{j} v_{j}^{-1}, \kappa^{-1}\left(\partial \Phi / \partial v_{k}\right) \Phi^{-1} \\
& =\tilde{c}_{k} v_{k}^{-1}-2\left(\tilde{y}_{k-1}+\beta_{k-1} \sigma_{k-1}\right)\left(v_{k}+1\right)^{-1}+\tilde{\sigma}_{k-1}\left(v_{k}+1 / 2\right)^{-1}(\eta-1),
\end{aligned}
$$

where $j \neq k, \eta=1$ for $A_{n-1}$ (i.e. for (12A)), $\eta=2$ in case $B_{n}$ (for (12)) and $\tilde{c}_{j}=-2 \sum_{i \geq j} \tilde{y}_{i}$. This system can be solved independently in the spaces $M_{s}^{\mu}=$ $\boldsymbol{C} f_{s}^{0}+\boldsymbol{C} f_{\sigma s}^{0}, s \in \boldsymbol{S}_{n}, s \geq \omega_{\mu}, l(\sigma s)=l(s)+1$, where $\sigma=\sigma_{k-1}$.

Lemma 1. Put $v_{+}=-u_{s^{-1}(k)}, \quad v_{-}=-u_{s^{-1}(k-1)}, \quad w=-4 v_{k}\left(v_{k}+1\right), \quad \delta=$ $v_{+}-v_{-}, g_{1}^{ \pm}=1, g_{2}^{+}=-1, g_{2}^{-}=(\delta-1) /(\delta+1)$.

There are two linearly independent solutions $\varphi^{+}, \varphi^{-}$of (16) in $M_{s}^{\mu}$ with respect to the base $\left\{e_{1}, e_{2}\right\}$ :

$$
\begin{aligned}
e_{1} & =f_{\sigma s}^{0}+(\delta-1) \delta^{-1} f_{s}^{0}, e_{2}=f_{\sigma s}^{0}-(\delta+1) \delta^{-1} f_{s}^{0}, \\
\left(\varphi^{+}, \varphi^{-}\right) & =\left(\prod_{j \neq k} v_{j}^{\kappa \tilde{c}_{l}}\right) v_{k}^{\kappa \tilde{c}_{h+1}}\left(\begin{array}{c}
\varphi_{1}^{+} \varphi_{1}^{-} \\
\varphi_{2}^{+} \varphi_{2}^{-}
\end{array}\right), \\
\varphi_{l}^{ \pm} & =\left(2 v_{k+1}+1\right)^{(\eta-1)+l-1} w^{\eta \kappa v_{ \pm}} g_{l}^{ \pm} F\left(a^{ \pm}, b, c^{ \pm} ; w\right), l=1,2,
\end{aligned}
$$

where $F$ is the hypergeometric function with $a^{ \pm}=1 / 2 \pm \eta \kappa \delta / 2, b=\kappa \eta(1+\delta) /$ $2+l-1, c^{ \pm}=1 \pm \eta \kappa \delta, \tilde{c}_{j}(j \neq k)$ act on $M_{s}^{\mu}$ as some constants (see above).

To finish the proof of Theorem 3 we use the theory of Young bases for $\mathcal{H}_{n}^{q}$ from $[15,16]$. One can start from $F_{s_{h}}(u)=\left(T_{k}+\left(q-q^{-1}\right)\left(q^{2\left(u_{k}-u_{k+1}\right)}-1\right)^{-1}\right.$ $g\left(u_{k}-u_{k+1}\right)$ in place of $f_{s_{h}}$, where $q=\exp (\pi i \kappa), g(v)$ is any scalar function $(g(0) \neq 0)$. We construct $\left\{F_{s}(u)\right\}$ by the same cocycle relation (15) as for $\left\{f_{s}\right\}$ and define $\left\{F_{s}^{0}, s \geq \omega=\omega_{\mu}\right\}$ for the same set $\hat{u}_{\mu}^{0}$. The properties of these bases 
were stated in [15-17]. We will consider $\boldsymbol{H}_{n}^{q}$ (of type $A_{n-1}$ ) as the subalgebra of $\mathscr{H}_{n}^{q}$ generated by $\left\{T_{1}, \ldots, T_{n-1}\right\}$.

Proposition 5. 1) Let us denoted by $\mathcal{M}_{u^{0}}$ the $\mathscr{H}_{n^{q}}^{q}$-module $H_{n}^{q}$ with the regular left action of $H_{n}^{q} \subset \mathcal{H}_{n}^{q}$ and the action of $\left\{Y_{k}, 1 \leq k \leq n\right\}$ defined by the relations $Y_{k}(1)=q^{2 u_{k}^{0}}$. Then $M_{u^{0}}$ contains $\mathcal{M}^{\mu} \stackrel{\text { def }}{=} \bigoplus_{s \geq \omega} C F_{s}^{0}$ as an irreducible $\mathcal{H}_{n}^{q}$-submodule (for generic $q$ ); $Y_{k}\left(F_{s}^{0}\right)=q^{2 u_{k}^{0}} F_{s}^{0}$ for $k^{\prime}=s^{-1}(k), 1 \leq k \leq n$. Any irreducible representation of $\mathscr{H}_{n}^{q}$ with semi-simple action of $\left\{Y_{k}\right\}$ has the form $\mathcal{M}^{\mu}$ for some $\mu$.

2) The modules $\mathcal{M}^{\mu}$ for $\mu$ with the only non-empty diagram $\mu^{1}$ (in case $A$ ) or with only two such diagrams $\mu^{+1}, \mu^{-1}$ (in case $B$ ) and with $d( \pm 1)=0$ constitute the set of all irreducible representations for the Hecke algebra $\mathbb{H}_{n}^{q}$ of type $A_{n-1}$ or for $\mathbb{H}_{n}^{q, \tilde{q}}$ of type $B_{n}$ with $\tilde{q}=\exp (\pi i \kappa \chi)$ (see Theorem 2 , where $Y_{n}^{-1}$ is to be taken as the image of $s_{n}$ ).

\section{Lemma 1 leads to}

Corollary 3. Put $g(x)=\exp (2 \pi i \kappa x) \Gamma(\kappa(x+1)) \Gamma \kappa(x-1)) \cdot \Gamma^{-2}(\kappa x)\left(x^{2}-1\right) x^{-2}$ for generic $\kappa$. Then the mapping $F_{s}^{0} \rightarrow f_{s}^{0}, s \geq \omega$ is extended to an isomorphism $M^{\mu} \rightarrow M^{\mu}$ of modules under the action of $\mathscr{H}_{n}^{q}$ and $\widetilde{\mathscr{H}}_{n}^{\prime}\left(\right.$ or $\left.\mathscr{H}_{n}^{\prime}\right)$ with respect to the homomorphisms $\widetilde{\beta}$ : $\mathscr{H}_{n}^{q} \rightarrow \widetilde{\mathscr{H}}_{n}^{\prime}$ (or its analogue $\beta$ for $\mathscr{H}_{n}^{\prime}$ ) from Theorem 3.

This corollary generalizes the main theorem of [6] and is connected with formulas from $[2,4]$. The usage of $\left\{Y_{k}\right\}$ and our appropach to Young bases make the construction as explicit as possible (cf. Theorem 5.8 from [4]).

It follows from the corollary that $\beta, \beta$ are isomorphisms (for general $\kappa$ ) and that formulas (14) are valid.

Concluding remarks. 1) One can construct some invariants of links by means of the results of this paper. Any link in $\mathbb{R}^{3} \backslash S^{1}$, where $S^{1}=\{t \in \mathbb{C},|t|=$ $1\}$, can be represented by an element (a braid) from the generalized braid group $\widetilde{B}_{n}$ in case $B_{n}, C_{n}$ for some $n$ (see (3), Section 1). The construction of general invariants of these links is similar to that of [18]. The simplest analogs of Jones polynomials use the trace $\operatorname{Tr}_{n}^{N}$ of the Hecke algebra $H_{n}^{q}$ acting on the tensor product of $n$ copies of some vector space $\mathbb{C}^{N}$ in the usual manner (Baxter, Jimbo).

We number the generators $s_{1}, s_{2}, \ldots, s_{n}$ from the formula (3) to ensure the validity of the relation $s_{1} s_{2} s_{1} s_{2}=s_{2} s_{1} s_{2} s_{1}$ and the relations $s_{i} s_{i+1} s_{i}=s_{i+1} s_{i} s_{i+1}$ $(i>1)$. Then $s_{1}$ corresponds to the passage of the first strand through $S^{1}, s_{i}$ is the permutation of the $i$-th strand and the $(i+1)$-th one. To construct the invariant one has to use the homomorphism $\beta_{m}: \widetilde{B}_{n} \rightarrow\left(H_{n+m}^{q}\right)^{*}$ taking $s_{1}$ to $T_{m} \ldots T_{2} T_{1}^{2} T_{2}$ $\ldots T_{m}$, braids $s_{i}$ to $T_{m+i-1}(1<i \leq n)$ and put $\left.\chi(s)=\operatorname{Tr}_{n+m}^{N}\left(M^{\otimes(n+m}\right) \beta_{m}(s)\right)$, $s \in \widetilde{B}_{n}, M=\operatorname{diag}\left(q^{N-1}, q^{N-3}, \ldots, q^{1-N}\right)$. This $\chi$ does not depend on $n$ (after a 
proper normalization - see e.g. [18]) and gives some Markov invariant for $\widetilde{\boldsymbol{B}}_{\infty}$ with the parameters $m, N, q$.

2) The equations (1) for general $S_{n}$-invariant $r$-matrices can be interpreted by means of some generalization of Kac-Moody algebras [5,19]. The function $\Phi$ turns out to be a version of the so-called $\tau$-function. In case of Yang's $r$-matrix this interpretation is closely connected with the primary fields from the conformal field theory (see $[1,2])$.

3) The isomorphisms between Hecke algebras $\boldsymbol{H}_{n}^{q}, \mathscr{H}_{n}^{q}$ and their degenerations give some isomorphisms of the corresponding quantum groups for general $q$ and the universal enveloping algebras or between the yangians and their $q$-analogs from [16].

4) The author thanks the participants of the A.N. Varchenko-D.B. Fuks seminar in Moscow State University, I.M. Gelfand for their kind interest in this work, T. Tanisaki for several important remarks and especially T. Miwa for useful discussion and advice. He is grateful for the kind invitations to the Hayashibara Forum 90 on special functions (Okayama 1990) and to the RIMS seminars, where this work was reported.

Its preliminary version was published as preprint ITF 89-74E (Kiev, 1989) with the same title. Some continuation is in paper [20] and in the Proceedings of the Okayama conference.

\section{References}

[1] Knizhnik, V.G. and Zamolodchikov, A.B., Current algebra and Wess-Zumino models in two dimensions, Nucl. Phys., B247 (1984), 83-103.

[2] Tsuchiya, A. and Kanie, Y., Vertex operators in two dimensional conformal field theory on $P^{1}$ and monodromy representation of braid groups, Adv. Stud. in Pure Math., 16 (1988), 297-372.

[3] Kohno, T., Monodromy representations of braid groups and Yang-Baxter equations, Ann. Inst. Fourier. Grenoble, 37 (1987), 139-160.

[4] , Quantized universal enveloping algebras and monodromy of braid groups, Preprint series No. 3, 1989.

[5] Cherednik, I.V., Generalized braid groups and local $r$-matrix systems, Doklady Akad. Nauk SSSR, 307 (1989), 27-34.

[6] On a calculation of the monodromy for some $W$-invariant local systems of type B, C, D, Funct. Anal. Appl., 23 (1989), 91-92.

[7] Bourbaki, N., Groups et algebres de Lie, Chapitre 6, Hermann, Paris, 1969.

[8] Cherednik, I.V., Factorizing particles on the half-line and root systems, Theor. Math. Phys., 12 (1971), 91-92.

[9] Brieskorn, E., Die Fundamentalgruppe des Raumes der regulären Orbits einer endlichen Kompexen Spiedelungsgruppe, Inventiones Math., 1 (1971), 57-61.

[10] Coddington, E.A. and Levinson, N., Theory of ordinary differential equations, McGraw-Hill, 1955.

[11] Lusztig, G., Some examples of square integrable representations of semisimple $p$-adic groups, Trans. Am. Math. Soc., 277 (1983), 623-653.

[12] Rogawski, J.D., On modules over the Hecke algebra of a p-adic group, Invent. Math., 79 (1985), 443-465. 
[13] Zelevinsky, A.V., Induced representations of reductive $p$-adic groups II, Ann. Sci. E.N.S., 13 (1980), 165-210.

[14] Cherednik, I.V., On special bases of irreducible representations of the degenerated affine Hecke algebra, Funct. Anal. Appl., 20 (1986), 87-89.

[15] On $R$-matrix quantization of formal loop groups, In: Group Theoretic Methods in Physics. Proceed. of the third seminar, Yurmala, 1985 (Nauka, Moscow, 1986. VNU Publ. B.V., 1986).

[16] — A new interpretation of Gelfand-Tzetlin bases, Duke Math. J., 54 (1987), 563-577.

[17] Dipper, R. and James, G.D., Blocks and idempotents of Hecke algebras of general linear groups. Proc. London Math. Soc., 54 (1987), 57-82.

[18] Reshetikhin, N.Y., Quantized universal enveloping algebras, the Yang-Baxter equation and invariants of links I, II, LOMI Preprint E-4-87, E-17-87, Leningrad, 1988.

[19] Cherednik, I.V., Functional realizations of basic representations of factorizable groups and Lie algebras, Funct. Anal. Appl., 19 (1985), 36-52.

[20] - A univormization of Knizhnik-Zamolodchikov and Dunkl operators via affine Hecke algebras, RIMS Preprint, 724, Kyoto, 1990. 